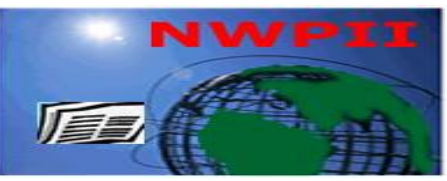

American Journal of Biomedical Sciences

ISSN: 1937-9080

nwpii.com/ajbms

\title{
A Broad Evaluation of Left Breast Radiotherapy
}

\begin{abstract}
Aime M Gloi
Radiation Oncology- 21st Century Oncology of California, USA

"Corresponding Author

Aime M Gloi

21st Century Oncology of California

1316 Nelson Avenue, Modesto, CA 95350

USA

Phone:209-575-5870

Fax:209-575-5872

Email: agloi7288@gmail.com

Received:16 April 2019; | Revised:04 May 2019; | Accepted: 04 September 2019

Abstract

Background: The aim of this study was to evaluate radiobiological model parameters and the secondary cancer risk to patients undergoing hypofractionated or conventionally fractionated irradiation of the left breast. Materials and Methods: 17 conventional (50 Gy) and 13 hypofractionated (42.56 Gy) plans were randomly selected and both biologically equivalent dose (EQD2) and effective dose (BED) were assessed in addition to dose homogeneity index, equivalent uniform dose, normal tissue complication probability (NTCP), and tumor control probability (TCP). Secondary cancer risks were estimated through dose volume histogram (DVHs) by calculating organ equivalent doses (OED) and excess absolute risks (EAR) using linear, bell-shaped, and plateau models. Results: EQD2 and TCPs were steady throughout a spectrum of a / $\beta$ ratios. TCPs for conventional treatment are $84.36 \pm 7.68,99.30 \pm 0.71$, and $17.87 \pm 6.92$ for $\alpha / \beta=4$, $\alpha / \beta=2.5$, and $\alpha / \beta=10$ respectively. Likewise, the TCP values for the hypofractionated regimen are $91.04 \pm 11.43,98.67 \pm 4.23$, and $24.31 \pm 8.74$ for $\alpha / \beta=4, \alpha / \beta=2.5, \alpha / \beta=10$ respectively. NTCP based LKB model with the pericarditis endpoint yielded a $0 \%$ risk. Logistic regression and LKB model have similar mean lung dose and can be used interchangeably for lung pneumonitis. A combined heart-lung, cardiac failure, and logistic lung model, based on this study, discloses a higher probability of heart failure in conventional treatment and lung toxicity in hypofraction. Estimates of secondary cancer were higher for conventional plans compared to hypofraction for all models, for patients over 30 years old. Conclusions: This work reveals significant similarities and differences between the hypofractionated and conventional treatment of the left breast, via radiobiology.
\end{abstract}

Keywords: Equivalent uniform dose (EUD), Normal tissue complication probability (NTCP), Tumor control probability (TCP), Organ-equivalent dose (OED), Excess absolute risk (EAR) 


\section{Introduction}

Breast-conserving therapy (BCT) has long been the optimal treatment for patients with earlystage breast cancer. It involves surgical removal of the tumor, followed by a histological assessment of the axillary lymph nodes, and finally adjuvant radiotherapy to the whole breast with the possibility of an additional boost to the tumor bed. Conventional breast irradiation therapy applies a daily 2 Gy fraction over 5 to 6 weeks of treatment. Such a lengthy treatment schedule is detrimental to the patient's quality of life and financial resources. However, new randomized trials ${ }^{[1-5]}$ have shown that other regimens such as hypofractionation have survival and local control outcomes equivalent to conventional therapy, and similar or superior acute toxicity outcomes ${ }^{[6-7]}$. Therefore, hypofractionated (2.66 Gy $\times 16$ fractions) regimens are now widely used, with secondary radiobiological outcomes different from conventional breast treatment. The design uses a higher dose per fraction, so the linear - quadratic (LQ) model used in conventional radiotherapy is difficult to extend to the hypofraction scheme. Both treatment schedules assume that reoxygenation happens in hypoxic tumor regions and that these regions benefit from the repair capacities of adjacent late-responding normal tissue ${ }^{[8]}$.

Recent studies in the United Kingdom provide valuable information on the effectiveness of breast cancer hypofractionation ${ }^{[9-10]}$. They describe a clinical trial with 1,234 patients divided into two subgroups: 622 patients received 42.56 Gy in 16 fractions, and 612 patients received 50 Gy in 25 fractions. Tissue fractionation sensitivities reported by the study were in the range of $3-4 \mathrm{~Gy}$, comparable to normal tissue and indicating a relatively large proportion of repairable radiation lesions ${ }^{[11]}$. Based on their findings, they suggest specific a / $\beta$ ratios to characterize tissue radiosensitivities corresponding to the possible outcomes of breast irradiation: $\alpha / \beta=4$ for tumor control, $\alpha / \beta=2.5$ for fibrosis, and $\alpha / \beta=10$ for erythema.

This study presents a comprehensive radiobiological analysis of conventional and hypofractionated therapies on thirty patients who were treated for an early-stage tumor in the left breast. We calculate three-dimensional (3D) dose distributions and differential dose volume histograms (dDVH). The goal of any radiation therapy is to maximize the tumor control probability (TCP) and minimize normal tissue complication probability (NTCP) for each organ at risk. We also evaluated other common treatment metrics such as equivalent uniform dose (EUD), dose homogeneity index (DHI), S-index, and quality factor (QF). We assessed the risk of long-term toxicities, particularly damage to the cardiorespiratory system and radiation-induced second cancer, by means of a predictive model. We chose these risks not only because left lung and heart tissue are close to the left breast, but also because newer radiation therapy techniques such as intensity modulation radiotherapy (IMRT) and volumetric modulated arc therapy (VMAT) can bathe a large region of lung tissue in a low dose. Note that unrelated factors not controlled in this study, such as age, social habits, and pre-existing conditions, may also contribute to heart and lung radiation-induced toxicity.

\section{Materials and Methods}

The study was performed on two groups of patients with cancer of the left breast, who were treated with external beam radiotherapy. Thirteen patients received 25 daily 2.0 Gy fractions for a total dose of $50 \mathrm{~Gy}$, and five patients received 28 daily $1.8 \mathrm{~Gy}$ fractions for a total dose of $50.4 \mathrm{~Gy}$. These 18 patients are designated the "conventional" group. Thirteen patients received 16 daily $2.66 \mathrm{~Gy}$ fractions for a total dose of 42.56 Gy and are called the "hypofractionated" group. All patients were scanned using computed tomography (CT) GE Hispeed CT-scanner (GE Medical Systems, USA), in the supine position with their arms over their heads. The target volume (PTV) and the organs at risk (OAR) were contoured, and treatment plans were generated, using the Eclipse (Varian Medical Systems, Palo Alto, USA) treatment planning system (TPS). Differential dose volume histogram (dDVH) data were exported from the Eclipse TPS in ASCII format, for a dose bin size of $20 \mathrm{cGy}$. These data were imported into other software (Bioplan ver.1.3.3) ${ }^{[12]}$ for the calculation of EUD, NTCP, and TCP. 


\subsection{DVH metrics}

The quality of a treatment plan is measured through its dose volume histogram (DVH) which offers a true account of dose homogeneity across the PTV. The dose homogeneity index (DHI), a summary statistic of the DVH, is a good indicator of the shape of the dose distribution within a target volume. (Roughly, a higher value of DHI means that the transition region from high to low dose covers a larger volume.) DHI can be used as a convenient metric to compare different treatment plans. It is calculated as follows:

$$
D H I=\frac{D_{2 \%}-D_{98 \%}}{D_{50 \%}}
$$

where $\mathrm{D} 2 \%$ represents the dose delivered to $2 \%$ of the PTV, D98\% denotes the dose delivered to $98 \%$ of the PTV, and D50\% is the $50 \%$ prescribed dose.

We also calculate the sigma-index ("S-index") ${ }^{[13]}$ of the DVH, which evaluates the uniformity of the delivered dose over the target volume. The Sindex is defined as

$$
S_{\text {index }}=\sqrt{\sum_{i}\left(D_{i}-D_{\text {mean }}\right)^{2} \times \frac{v_{i}}{V_{T}}}
$$

where Di is the delivered dose to the $\mathrm{i}$-th voxel, vi is the corresponding volume, Dmean is the mean dose, and VT is the total target volume.

Quality Factor (QF)

Another commonly used dosimetric index is the Quality Factor (QF) ${ }^{[14]}$, which assesses the overall plan and encompasses all indices affiliated with the plan. The QF of a plan is given analytically by:

$$
Q F=\left[2.718 \times e^{\left(-\sum^{n} W_{i} X_{i}\right)}\right]
$$

where the Xi represents all indices used for evaluating a plan (here, they are DHI and the Sindex), and the $\mathrm{Wi}$ are weighting factors. The weights Wi vary from 0 to 1 and are set to $1 / 2$ in our case of two indices.

\subsection{Radiobiological evaluation}

Niemierko and the Lyman-Kutcher-Burman (LKB) reduction scheme are commonly used to calculate NTCP, EUD, and TCP.

\subsubsection{Niemierko}

The phenomenological model proposed by Niemierko [15] for evaluating EUD (and subsequently, TCP and NTCP) from the dDVH is

$$
E U D=\left(\sum_{i=1}\left(v_{i} E Q D_{i}^{a}\right)\right)^{\frac{1}{a}}
$$

where a is a dimensionless constant specific to the normal or tumor tissue of interest, and vi is the partial volume corresponding to dose Di. EQD2 (henceforth EQD2) is a biologically equivalent dose of $2 \mathrm{~Gy}$, which can be derived from Equation (1) as follows:

$$
E Q D 2=D \times \frac{\left(\frac{\alpha}{\beta}+\frac{D}{n_{t}}\right)}{\left(\frac{\alpha}{\beta}+2\right)}
$$

where nt and $\mathrm{dt}=\mathrm{D} / \mathrm{nt}$ are the total number of fractions and the dose per fraction respectively. The ratio $\alpha / \beta$ is the tissue radiosensitivity.

We also used the mathematical concept of biologically effective dose (BED) to illustrate the biological effects observed after irradiation. It is derived from the LQ model and is defined as:

$$
B E D=n \times d \times\left[1+\frac{d}{\frac{\alpha}{\beta}}\right]
$$

where $\mathrm{n}$ is the number of fractions, $\mathrm{d}$ is the dose per fraction, and $\alpha / \beta$ is the ratio of the radiosensitivity coefficients.

Based on the EUD model, the tumor control probability (TCP) is given by:

$$
T C P=\frac{1}{1+\left(\frac{T C D_{50}}{E U D}\right)^{4 / 50}}
$$


TCD50 is the dose that controls $50 \%$ of tumors, assuming that the tumor is homogeneously irradiated. The parameter $\gamma 50$ describes the slope of the dose-response curve and is unitless. Using a logistic function, NTCP is obtained from the EUD as:

$$
N T C P=\frac{1}{1+\left(\frac{T D_{50}}{E U D}\right)^{4 \gamma 50}}
$$

where TD50 is the "tolerance dose" that yields a $50 \%$ probability of subsequent complications.

\subsubsection{Lyman-Kutcher-Burman (LKB) NTCP model}

We also fit the data to a LKB NTCP model ${ }^{[16-}$ ${ }^{18]}$, in order to assess the effects of radiation dose and the irradiated volume on the probability of radiation-induced changes in the left lung, total lung, and heart function. The LKB model is based on a sigmoid dose-response curve with three parameters: $\mathrm{n}, \mathrm{m}$, and TD50. The parameter $\mathrm{n}$ accounts for the volume effect of an organ. The parameter $\mathrm{m}$ describes the slope of the dose-response curve. TD50 is the dose for uniform irradiation of the whole or partial volume that results in a $50 \%$ probability of subsequent complications. The NTCP is calculated as follows:

$$
\begin{aligned}
& N T C P=\frac{1}{\sqrt{2 \pi}} \int_{-\infty}^{t} e^{\frac{x^{2}}{2}} d x \\
& t=\frac{E U D-T D_{50}}{m \bullet T D_{50}}
\end{aligned}
$$

We use TD50 $=29.20 \mathrm{~Gy}, \mathrm{n}=1, \mathrm{~m}=0.45$, and $\alpha / \beta=3$ for radiation-induced pneumonitis. We use TD50 $=48$ Gy, $\mathrm{n}=0.35$, and $\mathrm{m}=0.1$ and $\alpha / \beta$ $=3$ for radiation-induced pericarditis.

\subsubsection{Logistic function model (NTCP $P_{\text {logistic) }}$}

The logistic function model from Marks et al.

[19] was used to estimate pneumonitis risk for the lung. The association between pneumonitis risk and mean lung dose (MLD) is evaluated as:

$$
N T C P_{\log \text { istics }}=\frac{\exp \left(b_{0}+b_{1} \times M L D\right)}{1+\exp \left(b_{0}+b_{1} \times M L D\right)}
$$

where the parameters are: $b 0=-3.87$ and b1 $=0.126 \mathrm{~Gy}-1$

\subsubsection{Cardiac failure}

In order to evaluate the relationship between mean heart dose and the risk of cardiac failure, we use the linear-quadratic model from Guldner et al. [20]:

$$
O R=1+\alpha_{1} \times D+\alpha_{2} \times D^{2}
$$

where $\mathrm{D}$ is the mean heart dose, a $1=0.19$ and $\alpha 2=0.002$.

\subsubsection{LKB lung heart interface}

We assessed the combined heart and lung risks during radiotherapy using the fitting model proposed by Cella et al. [21]. This model is parameterized using a linear combination of heart maximum dose (HDmax) in Gy, heart volume (HVol) in cc, and left lung volume (LLVol):

$$
N T C P_{\text {lung-heart }}=\frac{1}{1+e^{-\varepsilon}}
$$

where $\varepsilon=0.14 \times$ HDmax $+0.01 \times$ Hvol $0.002 \times$ LLVol -5.65

\subsection{Risk models using the DVH}

In this study, radiation-induced cancer was evaluated using standards and assumptions received from the International Commission on Radiological Protection (ICRP) ${ }^{[22]}$ and the Biological Effects of Ionizing Radiation Committee (BEIR) ${ }^{[23]}$. These sources lead to several additional models derived from the dose volume histogram.

\subsubsection{The OED model}

The concept of organ-equivalent dose (OED) was first introduced by Schneider et al. ${ }^{[24]}$ in an effort to model of secondary cancer induction for radiotherapy patients. The principle of OED is that for doses below $2 \mathrm{~Gy}$, the secondary cancer risk follows a linear relationship with dose, similar to the linear no-threshold path (LNT) model. Later efforts to include the effects of higher doses include the plateau model and the linear-exponential "bellshaped" model. Using the DVHs, we estimated the cancer risk for the left lung in both groups of 
patients using a linear OED model and the two nonlinear models mentioned above:

$$
\begin{gathered}
O E D_{\text {inear }}=\frac{1}{V_{T}} \sum_{I} V_{i} \times D_{i} \\
O E D_{\text {Bell-Shaped }}=\frac{1}{V_{T}} \sum_{i} V_{i} \times D_{i} e^{-\alpha \times D_{i}} \\
O E D_{\text {plateau }}=\frac{1}{V_{T}} \sum_{i} V_{i} \times \frac{1-e^{-\delta \times D_{i}}}{\delta}
\end{gathered}
$$

In these models, $\alpha$ is a tissue-specific parameter and $\delta$ is an organ-specific dose-response parameter. For lung, we used the parameters a $=0.129$ for the linear exponential model and $\delta$ $=0.139 \mathrm{~Gy}-1$ for the plateau model.

\subsubsection{The excess absolute risk (EAR)}

The EAR (excess cancers per 10,000 personyears) is defined as the product of the OED and the initial slope of the dose-response curve in the lowdose region. The EAR can be obtained by a convolution of OED and patient age stage, in the form:

$$
E A R=\beta * O E D * \mu(\text { agex }, \text { agea })
$$

where agex represents the exposure age and agea is the attained age. The function $\mu$ modifies the population-specific risk, and is given as:

$$
\mu(\text { agex, agea })=\exp \left(\gamma_{e}(\text { agex }-30)+\gamma_{a} \ln \left(\frac{\text { agea }}{70}\right)\right)
$$

In this form, the fit parameters are genderaveraged and centered on an exposure age of 30 years and an attained age of 70 years. The initial slope $\beta$ and the parameters $\gamma$ e and $\gamma$ a are determined from a study of Japanese atomic bomb survivors ${ }^{[24]}$. For lung EAR in this study, we take $\beta=7.5, \gamma \mathrm{e}=0.002$, and $\gamma \mathrm{a}=4.23$.

\subsection{Statistical analysis}

Receiver operating characteristic (ROC) analysis and AUC (area under the curve) were used to assess the predictive power of NTCP derived from different models for organ at risk (lung, heart),
EUD, and OR as signs of lung pneumonitis and cardiac toxicity. For each set of NTCP, EUD, and OR, we designed logistic regression models to predict the emergence of lung pneumonitis and cardiac toxicity after radiation therapy of the left breast using hypofraction or conventional treatment. A ROC curve was then generated from a combination of that set and AUC calculated with a cut-off discriminant point was set at 0.5. A higher AUC indicates a more powerful predictor. An unpaired t-test was used to compare the treatment strategies. Results are considered significant at the $5 \%$ level $(\mathrm{p}<0.05)$.

\section{Results}

Patient characteristics are summarized in Table 1. The median ages were 63 years and 70 years for the conventional and hypofractionated treatment groups, respectively. All subjects were females with invasive ductal carcinoma. In this situation, follow-up care is vital to evaluate general health, look for any signs of new breast tumors that could be detected early, and examine treatment side effects up to six weeks later, in addition to weekly status checks to review skin toxicity. There is no standardized follow-up model for either treatment regimen; it is patient and doctor dependent. However, our clinic utilized the national comprehensive cancer network $(\mathrm{NCCN})$ guidelines in all cases.

\subsection{Coverage and plans quantifiers}

Figures $1 \mathrm{a}-\mathrm{b}$ show the isodose distribution and the dose-volume histogram (DVH) for one of the patients who underwent hypofractionated treatment. Figures 2a-b show the isodose distribution and DVH for one of the patients who underwent conventional treatment. The conventional plan clearly created a larger contiguous region receiving a high dose. The marked region in Figure 1a received $106.5 \%$ of the prescription dose within the PTV, while in Figure 2a received only 109\%. The dose homogeneity index (DHI) is close to 0.07 for both regimens. Based on a statistical analysis of the DVH, we derived the S-index for all patients to assess the dose homogeneity of the left breast treatment. The S-index provides an additional tool to characterize PTV homogeneity. The difference 
between the two regimens is more noticeable conventional treatment because DHI provides incomplete information about PTV homogeneity. Sindex and DHI are strongly correlated for both hypofractionated $\left(\mathrm{R}^{2}=0.967\right)$ and conventional $\left(\mathrm{R}^{2}=0.862\right)$ regimens, as illustrated in Figures $3 \mathrm{a}-\mathrm{b}$. These results also show that the S-index (Eq. 2) provides a better quantitative measure of the dose homogeneity than the DHI. In spite of the slightly higher DHI observed in conventional treatments, a lower QF (Eq. 3) value (recall that QF takes into account both DHI and the S-index) was observed. An unpaired t-test with Welch's correction was used to compare indices from both treatment regimens. There was no significant difference between the distributions of S-index $(p=0.0973)$ or DHI $(p=0.6359)$ when comparing the two regimens. However, the distributions of QF scores were significantly different $(p=0.0023)$; hence, we can conclude that overall, the hypofractionated regimen achieves better dose homogeneity.

Table 1: Patient characteristics

\begin{tabular}{|l|l|l|}
\hline Characteristic & Conventional & Hypofractionated \\
\hline $\mathrm{n}$ & 17 & 13 \\
\hline Age, median & 63 & 70 \\
\hline PTV $\left(\mathrm{cm}^{3}\right)$ & $1469.74 \pm 460.32$ & $804.33 \pm 303.24$ \\
\hline Heart & $444.97 \pm 96.98$ & $452.12 \pm 113.41$ \\
\hline Left Lung & $936.24 \pm 263.05$ & $916.51 \pm 139.00$ \\
\hline Total Lung & $2082.22 \pm 459.37$ & $2084.05 \pm 479.40$ \\
\hline Hot Spot & $109.05 \pm 1.66$ & $106.53 \pm 1.09$ \\
\hline DHI & $0.07 \pm 0.014$ & $0.07 \pm 0.04$ \\
\hline S_index & $3.30 \pm 0.63$ & $2.58 \pm 1.36$ \\
\hline
\end{tabular}

All reported values are mean \pm standard deviation.

\subsection{Radiobiology modeling (BED and EQD2)}

Table 2 compares the biologically effective dose (BED) and equivalent dose (EQD2) calculations for the two conventional regimens and one hypofractionated regimen. In general, the differences between the approaches were small but radiobiologically significant, indicating that further analysis is necessary. The weekly schedule consisting of 2.0 Gy per fraction has the highest clinical benefit for cancerous tissue in terms of BED (75 Gy) but also presents the highest effective dose relevant to the risk of fibrosis, a side effect which should be taken into account. The hypofractionated regimen of 2.66 Gy with 5 fractions seems safest in terms of the risk for developing acute erythema but is also associated with a greater risk of late fibrosis compared to the 1.8 Gy conventional regime. Unexpectedly, the conventional schedules seem to have the highest effective dose in terms of erythema. The study revealed that EQD2 dose-response relationship to be very steady throughout a range of $\alpha / \beta$ ratios in the setting of left breast conventional treatment.

Table 2: BED and EQD2 results from a variety of endpoints and radiosensitivities $(\alpha / \beta)$

\begin{tabular}{|l|l|l|l|}
\hline \multirow{2}{*}{$\begin{array}{l}\text { Treatment (dose per } \\
\text { fraction } \times \text { no. of }\end{array}$} & \multicolumn{3}{|c|}{ BED (Gy) and EQD2 (red) } \\
\cline { 2 - 4 } fractions) & Breast (tumor control): & Breast (late fibrosis): & Breast (Erythema): $\alpha / \beta=10$ \\
\hline Conventional $(2.0 \times 25)$ & $\alpha / \beta=4$ Gy & $\alpha / \beta=2.5$ Gy & 60 \\
& 50 & 90 & 50 \\
\hline Conventional (1.8x28) & 73.08 & 50 & 59.47 \\
& 48.72 & 86.69 & 49.56 \\
\hline Hypofraction $(2.66 \times 16)$ & 70.86 & 48.18 & 53.88 \\
& 47.24 & 87.84 & 44.9 \\
\hline
\end{tabular}




\subsection{Tumor Control Probability}

Table 3 lists the parameters for left breast irradiation under the conventional and hypofractionated regimens, with endpoints calculated using the Niemierko model. The TCPs (Eq. 6) are calculated using the Poisson model. The means and standard deviations of TCP for conventional left breast treatment in tissues with different radiosensitivities were $84.36 \pm 7.68$, $99.30 \pm 0.71$, and $17.87 \pm 6.92$ for $\alpha / \beta=4$ (tumor control), 2.5 (late fibrosis), and 10 (erythema) respectively. For the hypofractionated regimen, the TCPs were $91.04 \pm 11.43,98.67 \pm 4.23$, and $24.31 \pm 8.74$ for $\alpha / \beta=4,2.5$, and 10 respectively. In addition, Figures $4 \mathrm{a}-\mathrm{b}$ present correlation matrixes of the different TCP measures, in order to compare the effectiveness of a regimen in tissues with different radiosensitivities. For both treatment types, we found multiple strong correlations (i.e., Spearman's $r>0.638$ ) between the TCPs. It is clear from the above that the erythema endpoint has the lowest TCP in all treatment regimens. The average TCP for tumor control $(\alpha / \beta=4)$ is larger in the hypofractionated sample than in the conventional sample.

However, the hypofraction TCP is lower in the fibrosis $(\alpha / \beta=2.5)$ and erythema $(\alpha / \beta=10)$ regions. One can argue that these results can be separately changed based on histological grounds, clinical settings, and cytotoxic responses to external beam radiotherapy through the use of hormonal or chemotherapy treatment. We calculated coefficients of variation (CV), defined as the ratio of the standard deviation to the mean, to assess the relative variability within each radiosensitivity value and treatment regimen (Table 4). The erythema TCPs had the largest CVs, up to about 0.4. In addition, the radiosensitivity analysis reveals the impact of $\alpha / \beta$ on TCP values. Higher $\alpha / \beta$ ratios have a stronger dose effect, increasing the sensitivity to cold spots. This effect is acerbated by using different $\beta$ values from the linear-quadratic model. In fact, the model amplifies the importance of differences between low and high doses. When the a / $\beta$ ratio is lower, any contouring errors that could have been made on the PTV have less influence on the TCP. Estimates for a were commonly in the range of $0.02-0.2 \mathrm{~Gy}$ -1 , whereas $\beta$ values range from $0.001-0.06 \mathrm{~Gy}^{-2}$. To some extent, the $\beta$ values appear to be higher for breast tumors, translating into lower $\alpha / \beta$ values. In general, $\alpha$ / $\beta$ values are characterized by tumor site, whereas tumor response to radiation is governed by the tumor surroundings.

Table 3: Parameters used to calculate Niemierko's EUD-based TCP for left breast cancer and NTCP for left lung, total lung and heart

\begin{tabular}{|c|c|c|c|c|c|c|c|}
\hline Organ & $\mathrm{a}$ & \multicolumn{3}{|c|}{$\alpha / \beta$} & $\mathrm{TD}_{50}$ & $\gamma 50$ & $\mathrm{TCD}_{50}$ \\
\hline Breast PTV & -7.2 & $\begin{array}{c}4 \\
\text { Tumor } \\
\text { control }\end{array}$ & $\begin{array}{c}2.5 \\
\text { Late } \\
\text { fibrosis }\end{array}$ & $\begin{array}{c}10 \\
\text { Erythema }\end{array}$ & & 2 & 28 \\
\hline Total Lung & 1 & \multicolumn{3}{|c|}{3} & 24.5 & 2 & - \\
\hline Left Lung & 1 & \multicolumn{3}{|c|}{3} & 24.5 & 2 & - \\
\hline Heart & 2 & \multicolumn{3}{|c|}{3} & 50 & 3 & - \\
\hline
\end{tabular}

Table 4: Tumor control probability parameters and coefficient of variation for variable radiosensitivity for conventional and hypofraction of the left breast

\begin{tabular}{|c|c|c|c|}
\hline \multicolumn{4}{|c|}{ Conventional treatment } \\
\hline$\alpha / \beta$ & TCP mean & Std & CV \\
\hline 4 & 84.364 & 7.681 & 0.091 \\
\hline 2.5 & 99.305 & 0.710 & 0.007 \\
\hline 10 & 17.876 & 6.928 & 0.387 \\
\hline \multicolumn{4}{|c|}{ Hypofraction treatment } \\
\hline$\alpha / \beta$ & TCP mean & Std & CV \\
\hline 4 & 91.076 & 11.435 & 0.125 \\
\hline 2.5 & 98.669 & 4.231 & 0.042 \\
\hline 10 & 24.315 & 8.749 & 0.359 \\
\hline
\end{tabular}




\subsection{Organ at risk (OAR)}

Figure 5 shows the estimated risk of heart complications calculated from Eq. (11). The risk was considerably higher for conventional patients compared to hypofractionated patients. EUD and NTCP are commonly used as metrics for the biological effectiveness of a treatment regimen. Overall, this study observed a significant difference between hypofractionated and conventional regimens in terms of lung and heart risk. The NTCP calculated using the LKB model with the pericarditis endpoint $(\mathrm{m}=0.1$; TD50 $=48 \mathrm{~Gy} ; \mathrm{n}=$ $0.35 ; \alpha / \beta=3$ ) yielded $0 \%$ for both treatment regimens. In contrast, the dose-effect relationship between mean heart dose and cardiac failure resulted in different values for the conventional $(1.602 \pm 0.196)$ and hypofractionated $(1.301 \pm 0.148)$ treatments. There is a stronger correlation between EUDs and cardiac failure predictions under the hypofractionated regimen $\left(\mathrm{R}^{2}=0.9216, \mathrm{p}<0.0001\right)$ than under conventional treatment $(\mathrm{R} 2=0.892$, $\mathrm{p}<0.0001)$. The correlations are illustrated in Figures $6 a-b$. The difference between the two models is significant $(\mathrm{p}<0.0001)$, as attested by an unpaired t-test with Welch's correction. In addition, a major difference between the two regimens is that heart volume is poorly correlated with a cardiac complication in the hypofractionated sample $\left(\mathrm{R}^{2}=0.085\right)$, but strongly correlated in the conventional sample $\left(\mathrm{R}^{2}=0.958\right)$. Figure $7 \mathrm{a}-\mathrm{b}$ displays probability maps of the radiation-induced cardiac failure incidence in a Cartesian plane. The parameter space is EUD and heart volume, with patient ages displayed as interpolated contours. Hence, we see that older patients in a conventional treatment regimen have better EUDs and lower heart volumes. In addition, this map illustrates the complexity of correlating several parameters to standardize a viable model. To summarize, the dosimetric data for heart parameters such as OR, EUD, and normal tissue complication of the heartlung interface ( $\left.\mathrm{NTCP}_{\text {lung-heart }}\right)$ shows through Spearman's test shows that the strongest correlation is between the EUD and OR calculations $(\mathrm{R} 2=0.960)$, followed by the correlations between EUD and $\mathrm{NTCP}_{\text {lung-heart }}\left(\mathrm{R}^{2}=0.673\right)$ and $\mathrm{OR}$ and $\mathrm{NTCP}_{\text {lung-heart }}\left(\mathrm{R}^{2}=0.536\right)$. These results are for hypofractionated treatment of the left breast. For patients undergoing conventional left breast treatment, Spearman's test revealed similar correlations: the strongest between EUD and OR $\left(\mathrm{R}^{2}=0.944\right)$, followed by EUD and $\mathrm{NTCP}_{\text {lung-heart }}$ $\left(\mathrm{R}^{2}=0.648\right)$ and $\mathrm{OR}$ and $\mathrm{NTCP}_{\text {lung-heart }}\left(\mathrm{R}^{2}=0.538\right)$.

The mean NTCP percentage for left lung pneumonitis, calculated using the logistic function (Eq.10) $\mathrm{NTCP}_{\text {lung-logistic, showed a significant }}$ difference $(p<0.001)$ between the hypofractionated and conventional treatments. Figures 8 a-b assess the relationship between the LKB and logistic estimates of NTCP. Pearson's regression test reveals very strong correlations for both the hypofractionated $\left(\mathrm{R}^{2}=0.9946\right)$ and the conventional $\left(\mathrm{R}^{2}=0.9955\right)$ regimens. This result suggests that the NTCP-LKB (Eq.8) and NTCP-logistic (Eq.10) can be used interchangeably to estimate lung pneumonitis. In fact, when comparing the distributions of radiation doses delivered to the left lung for the 13 patients in the hypofractionated regimen and the 17 patients in the conventional regimen, we found no statistically significant difference. The average mean lung dose (MLD) was $3.24 \pm 0.67$ Gy in the hypofractionated sample vs. $5.28 \pm 1.93$ Gy in the conventional sample $(\mathrm{p}<$ 0.939, paired t-test). Furthermore, this analysis also demonstrates that the mean lung dose is adequate for evaluating lung pneumonitis.

To explore the interaction between the heart and lung parameters, a simple logistic model was used (Eq. 12). Figures 9a-b plot the anticipated $\mathrm{NTCP}_{\text {lung-heart }}$ from lung-heart irradiation against the NTCP probabilities for the heart (Eq. 11 for cardiac failure) and lung (Eq.10 for lung logistics) individually, for the hypofractionated and conventional treatments. As we can see, the two samples exhibit different patterns and also illustrate the previously described tendencies of the hypofractionated treatment towards higher lung NTCP (logistic estimate) and the conventional treatment towards cardiac failure (OR estimate). The data demonstrate that the hypofractionated sample exhibits a strong correlation between left lung EUD and both left lung NTCP $\left(\mathrm{R}^{2}=0.992\right)$ and total lung NTCP $\left(\mathrm{R}^{2}=0.998\right)$. The same is true for conventional treatment (left lung NTCP: $\mathrm{R}^{2}=0.983$, total lung NTCP: $\mathrm{R}^{2}=0.982$. In addition, Figures $10 \mathrm{a}-\mathrm{b}$ display correlation matrices summarizing the relationships between all DVH-based metrics, intraorgan (lung, heart) and inter-organ (left lung-heart) 
dosimetric variables, and TCPs calculated for various $a / \beta$ indices. For the hypofractionated treatment, we notice strong negative correlations between TCP for any $\alpha / \beta$ index and the DHI and S-index, but strong positive correlations between TCP and QF. Therefore, TCP can be characterized by $\mathrm{QF}$ for the hypofractionated regimen, in contrast with the conventional treatment.

In Figures 11a-b we investigate the quality of different NTCP models for predicting radiationrelated lung toxicity and heart complication. The quality of a model is evaluated using the area under the receiver operating characteristic (ROC) curve. Assuming a formal hypothesis $\mathrm{H} 0$ : AUC $=0.5$ versus $\mathrm{H1}$ : $\mathrm{AUC} \neq 0.5$ for this study, Table 5 shows

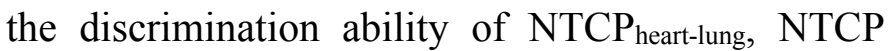
lung, and NTCP left lung ( $p$-value $<0.001$ ) for both hypofraction and conventional treatment, as well as EUD of the left lung ( $p$-value $>0.05$ ). For cardiac toxicity, EUD of the heart is a good test (pvalue $<0.0001$ ) for prediction in both treatment regimens.

Secondary cancer risk was estimated based on DVHs extracted from the TPS for all patients. We summarize the non-homogeneous organ dose distributions in high-dose regions using three different OED models, as described in the previous section. Figures $12 \mathrm{a}-\mathrm{b}$ present the calculated OEDs for the left lung. The mean OEDs ( \pm standard deviation) for conventional treatment were 58.12 \pm $19.28,11.41 \pm 1.99$, and $21.63 \pm 4.46 \mathrm{cGy}$ for the linear, bell-shaped, and plateau OED models respectively. Similarly, the mean OEDs ( \pm standard deviation) for hypofractionated treatment were $36.40 \pm 15.72,9.80 \pm 1.77,16.32 \pm 4.43 \mathrm{cGy}$ for the linear, bell-shaped, and plateau models respectively. The highest risk estimate is from the linear OED model in the conventional sample. Note that using an OED model to estimate the risk of radiation-induced cancer is comparable to using EUD to estimate TCP and NTCP. Figure 13 presents EAR estimates for inducing a secondary solid cancer as a function of attained age, for the different treatment types and risk models. We calculated results assuming that all patients had an age of 30 years at exposure and attained a final age of 70 years. For a given OED model, the conventional treatment always results in a higher EAR curve than the hypofractionated treatment, over all attained ages. The linear threshold OED model produces the highest EAR curve, whereas the bell-shaped model produces the lowest EAR curve. These results show that EAR is affected by both the choice of OED model and the treatment regimen. 30 years after treatment, the expected excess of secondary cancers in the hypofractionated sample is 135,60 , or 36 per 10000 patients for the linear, plateau, or bell-shaped OED models respectively. Similarly, the predicted excesses for the conventional sample are 215,80 , or 42 secondary cancers per 10000 patients for the linear, plateau, and bell-shaped OED models respectively.

Table 5: AUC estimates and 95\% confidence intervals of EUD and NTCP models for heart and lung for conventional and hypofraction treatment of the left breast

$\underline{\text { Conventional treatment }}$

\begin{tabular}{|l|l|l|l|l|l|}
\hline Model & AUC & Std. Error & P value & \multicolumn{2}{|c|}{ Lower \% CI for AUC } \\
& & & & & \\
\hline EUD Left Lung & 0.6332 & 0.1013 & 0.1848 & 0.4346 & 0.8318 \\
\hline NTCP $_{\text {heart-lung }}$ & 1.000 & 0.000 & $<0.0001$ & 1.000 & 1.000 \\
\hline NTCP Left Lung & 0.7647 & 0.08593 & 0.0084 & 0.5963 & 0.9331 \\
\hline NTCP Lung & 0.9931 & 0.009278 & $<0.0001$ & 0.9749 & 1.000 \\
\hline EUD Heart & 1.000 & 0.1001 & $<0.0001$ & 1.000 & 1.000 \\
\hline NTCP $_{\text {heart-lung }}$ & 1.000 & 0.1001 & $<0.0001$ & 1.000 & 1.000 \\
\hline
\end{tabular}


Hypofraction treatment

\begin{tabular}{|l|l|l|l|l|l|}
\hline Model & AUC & Std. Error & P value & \multicolumn{2}{|}{. 95\% CI for AUC } \\
& & & & & \\
\hline EUD Left Lung & 0.5089 & 0.1238 & 0.9387 & 0.2663 & 0.7514 \\
\hline NTCP $_{\text {heart-lung }}$ & 1.000 & 0.000 & $<0.0001$ & 1.000 & 1.000 \\
\hline NTCP Left Lung & 0.7574 & 0.09798 & 0.0257 & 0.5654 & 0.9494 \\
\hline NTCP Lung & 0.9941 & 0.00972 & $<0.0001$ & 0.9750 & 1.000 \\
\hline EUD Heart & 0.8462 & 0.1001 & 0.0027 & 0.6500 & 1.000 \\
\hline NTCP $_{\text {heart-lung }}$ & 1.000 & 0.000 & $<0.0001$ & 1.000 & 1.000 \\
\hline
\end{tabular}

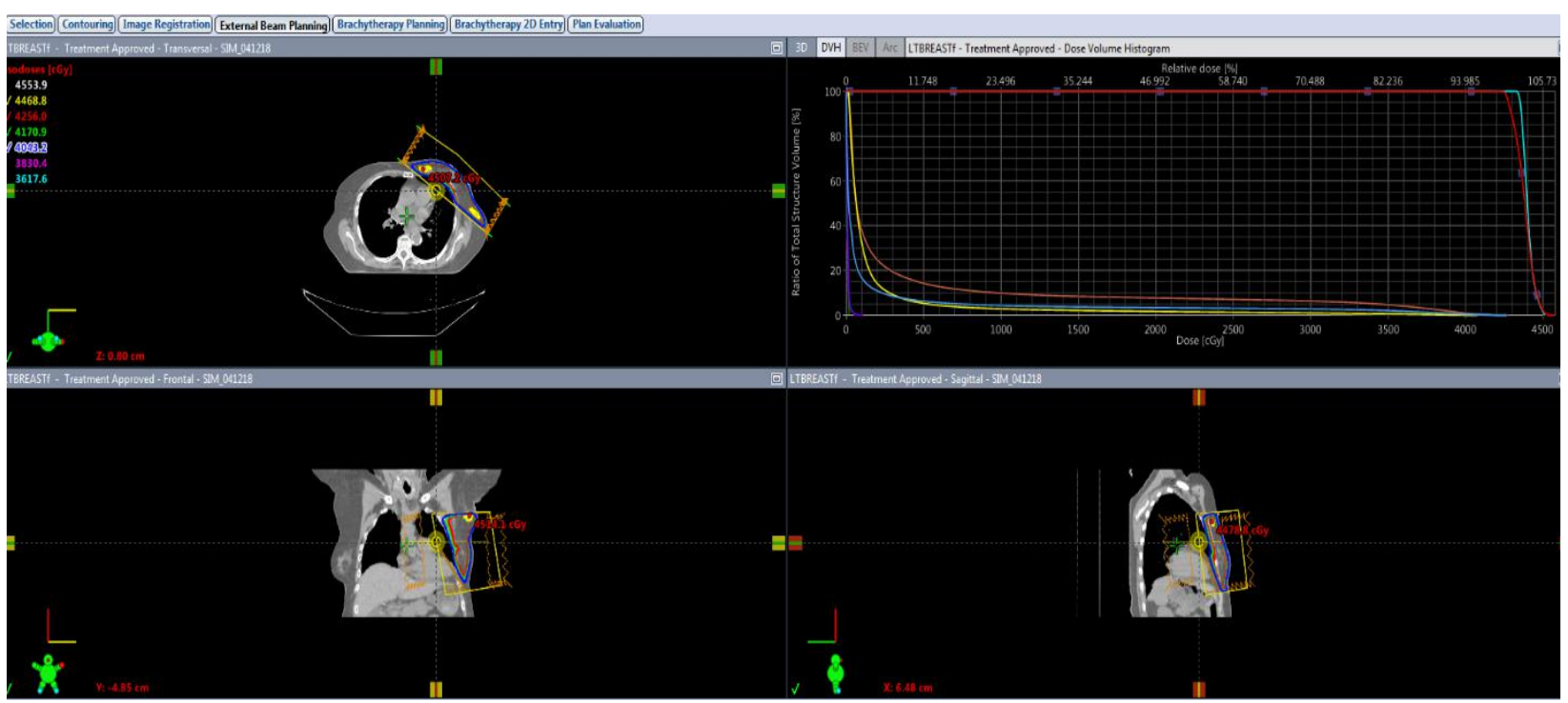

Figure 1a-b: Dose distributions and dose volume histograms of hypofraction left breast treatment

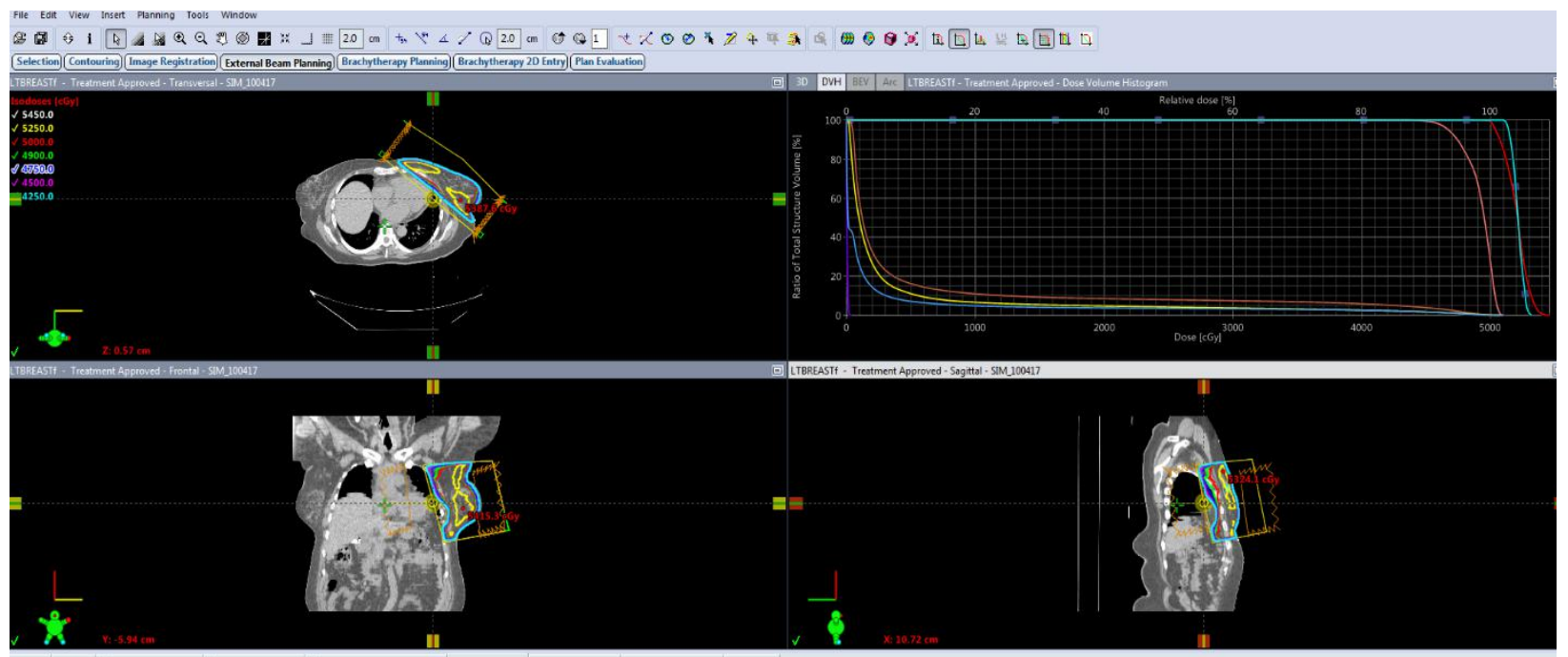

Figure 2 a-b: Dose distributions and dose volume histograms of conventional left breast treatment 


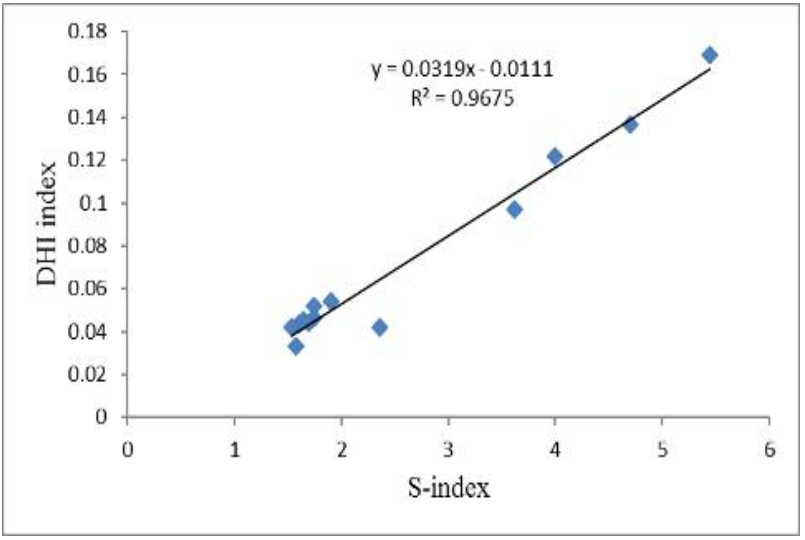

(a)

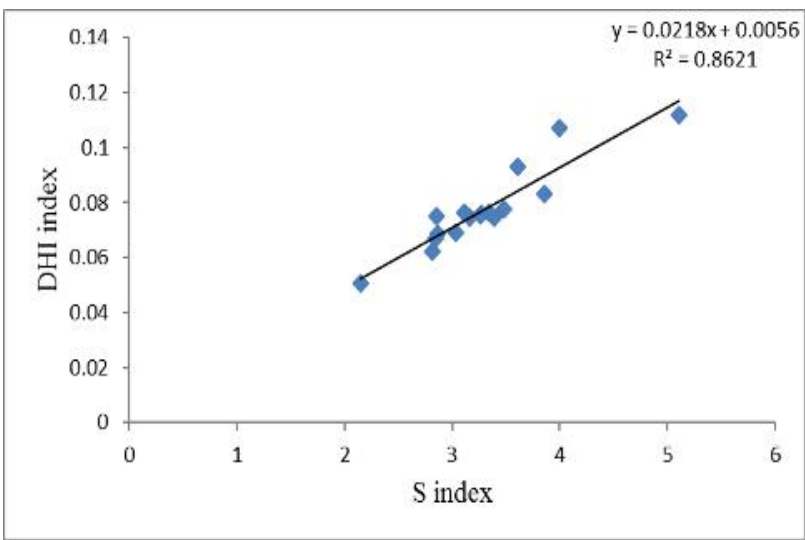

(b)

Figure 3a-b: The correlation between $S_{-}$index and DHI index for a) hypofraction and b) conventional left breast irradiation, respectively

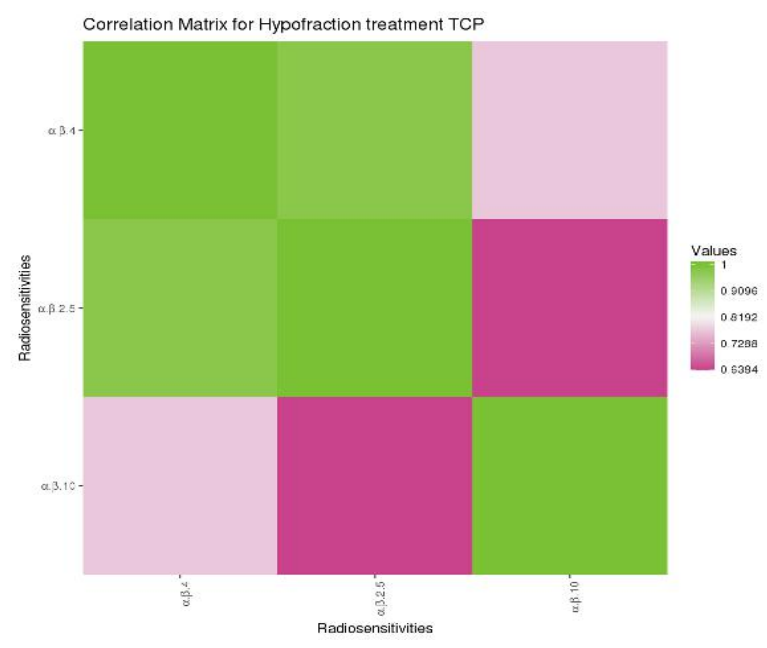

(a)

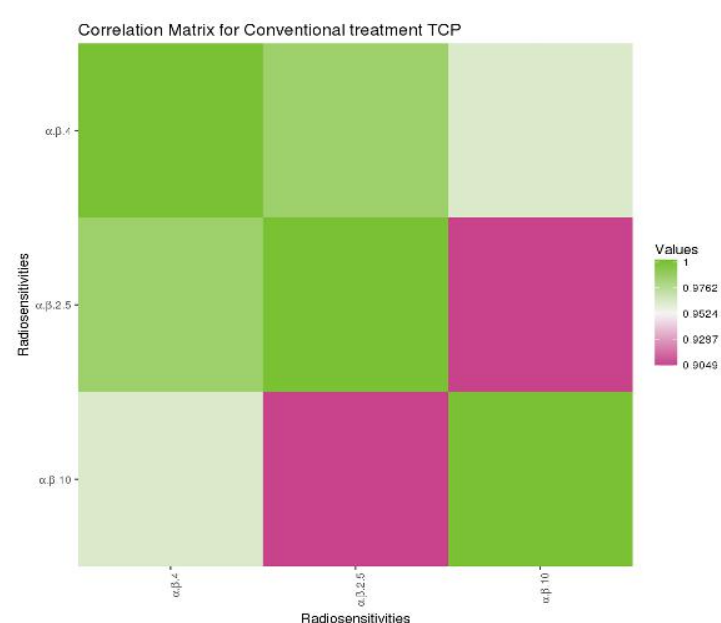

(b)

Figure 4a-b. The cross-correlation matrixes for TPC based radiosensitivities associated with hypofraction (a) and conventional treatment of left breast (b). The color-bar represents the Spearman's rank correlation coefficient value

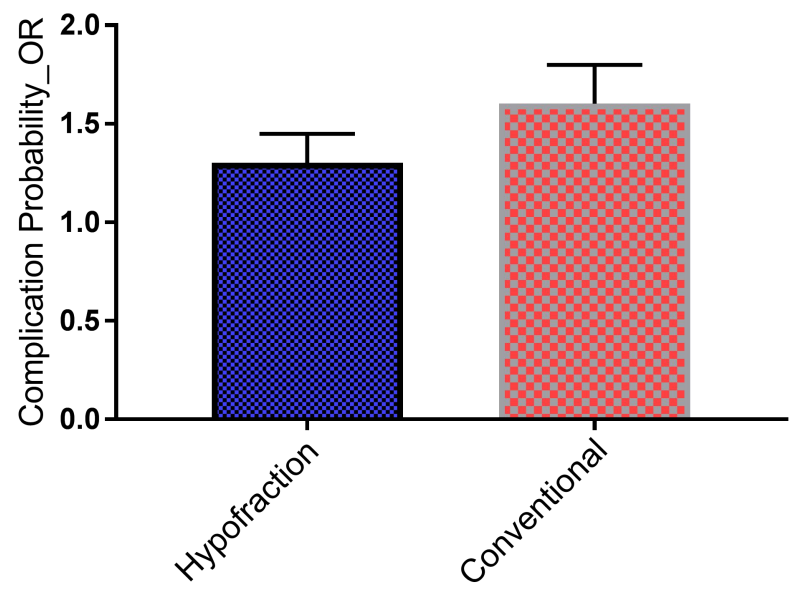

Figure 5: Mean and standard deviation values for patients comparing long-term risks of inducing heart failure between hypofraction and conventional fraction 


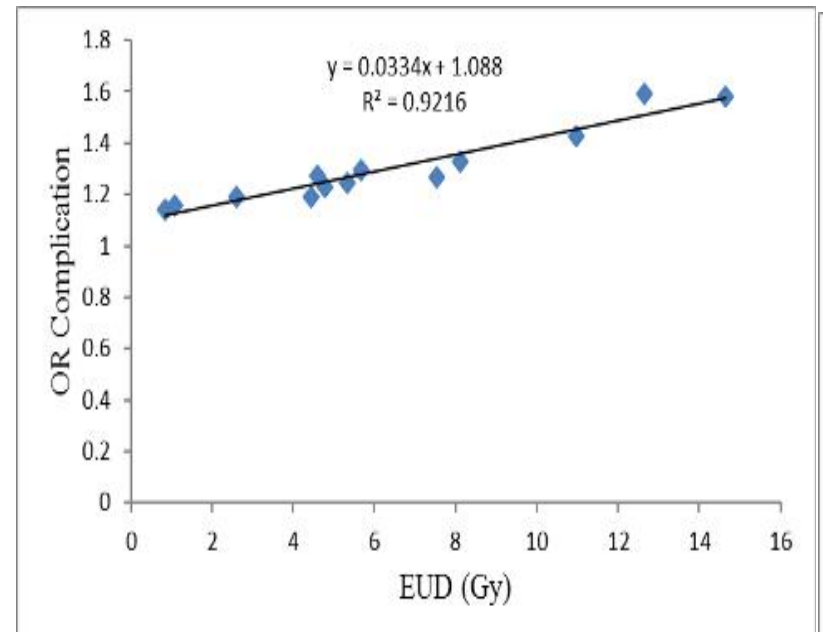

(a)

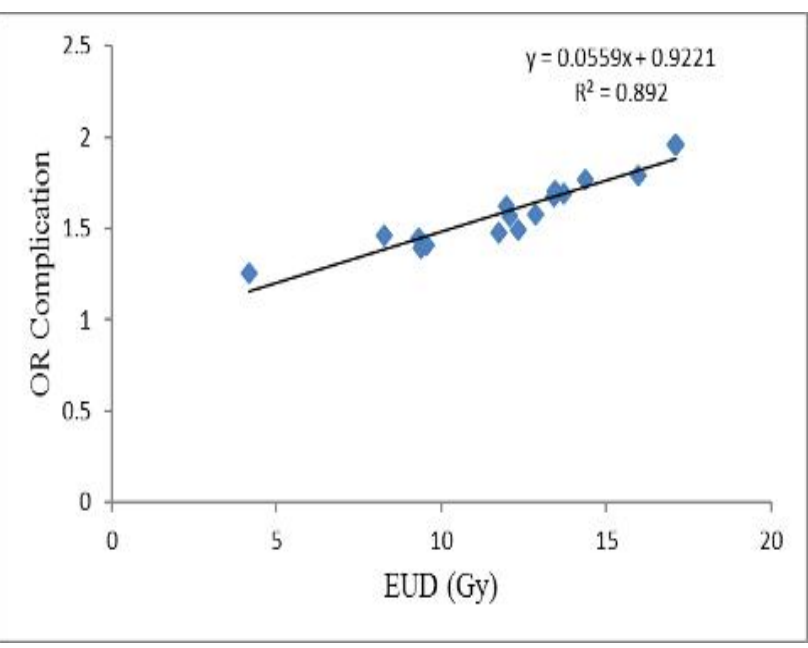

(b)

Figure 6 a-b: Correlation between mean heart dose cardiac failures and EUD for (a) hypofraction and (b) conventional left breast irradiation, respectively

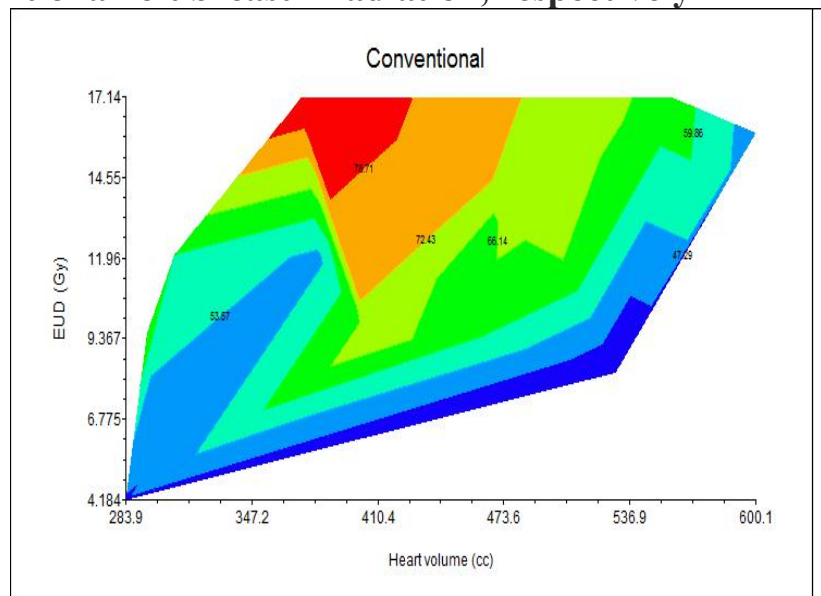

(a)

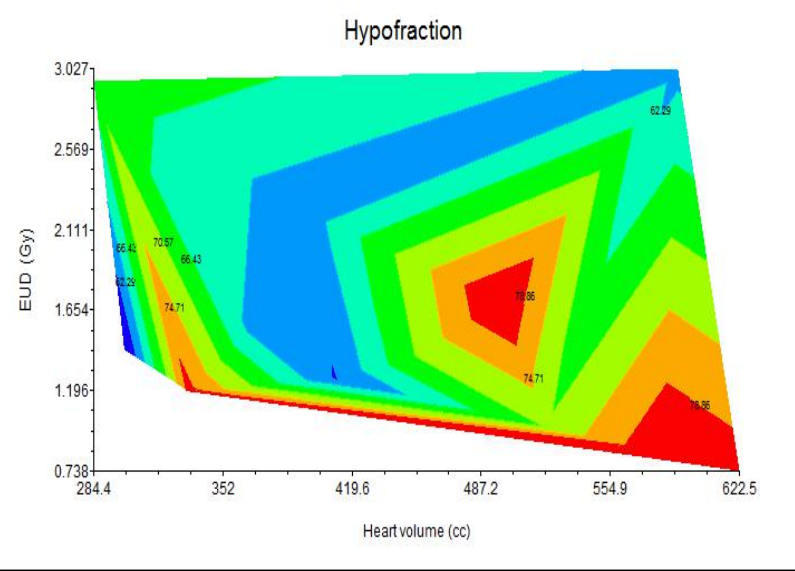

(b)

Figure 7 a-b: Illustration of radiation induced cardiac failure incidence probability maps in Cartesian plane centered on EUD, Heart volume, and Age for conventional (a) and hypofraction (b) for left breast patients

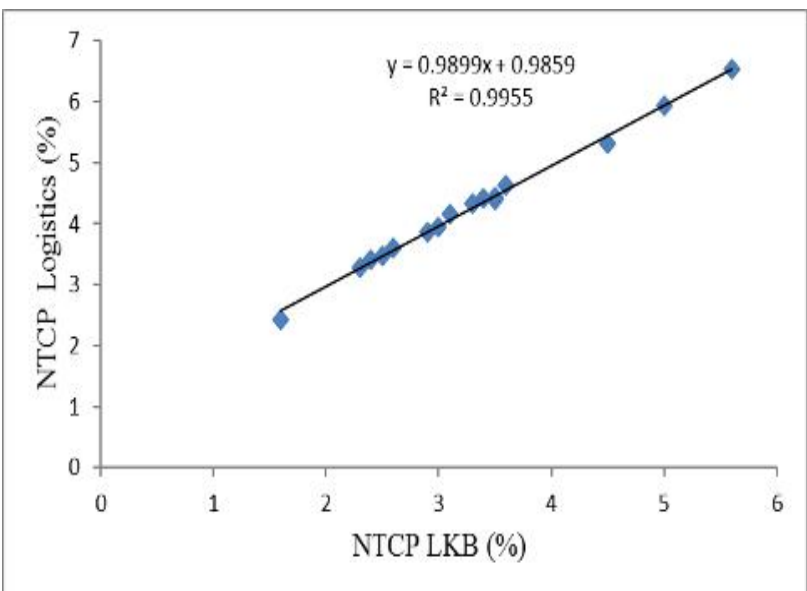

(a)

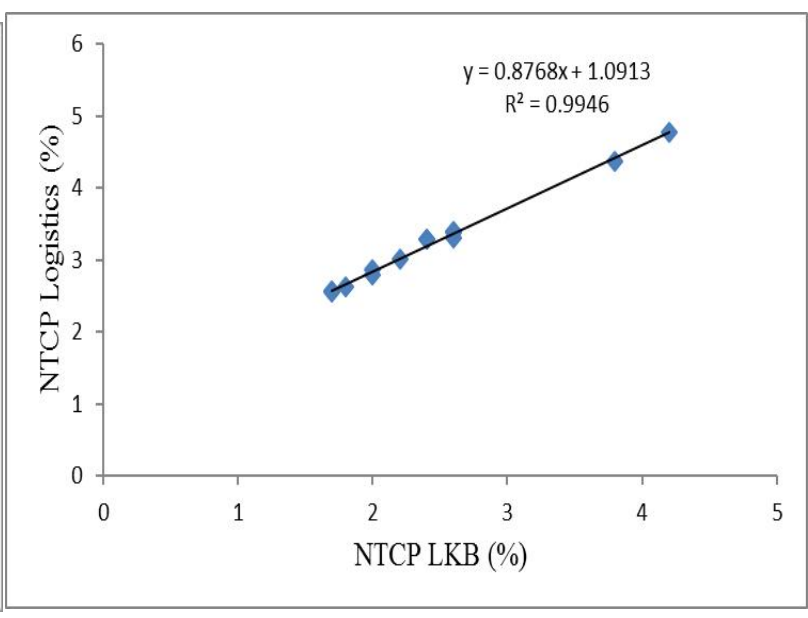

(b)

Figure 8 a-b: Correlation between NTCP logistics versus NTCP LKB for conventional (a) and hypofraction (b) of the left breast irradiation 


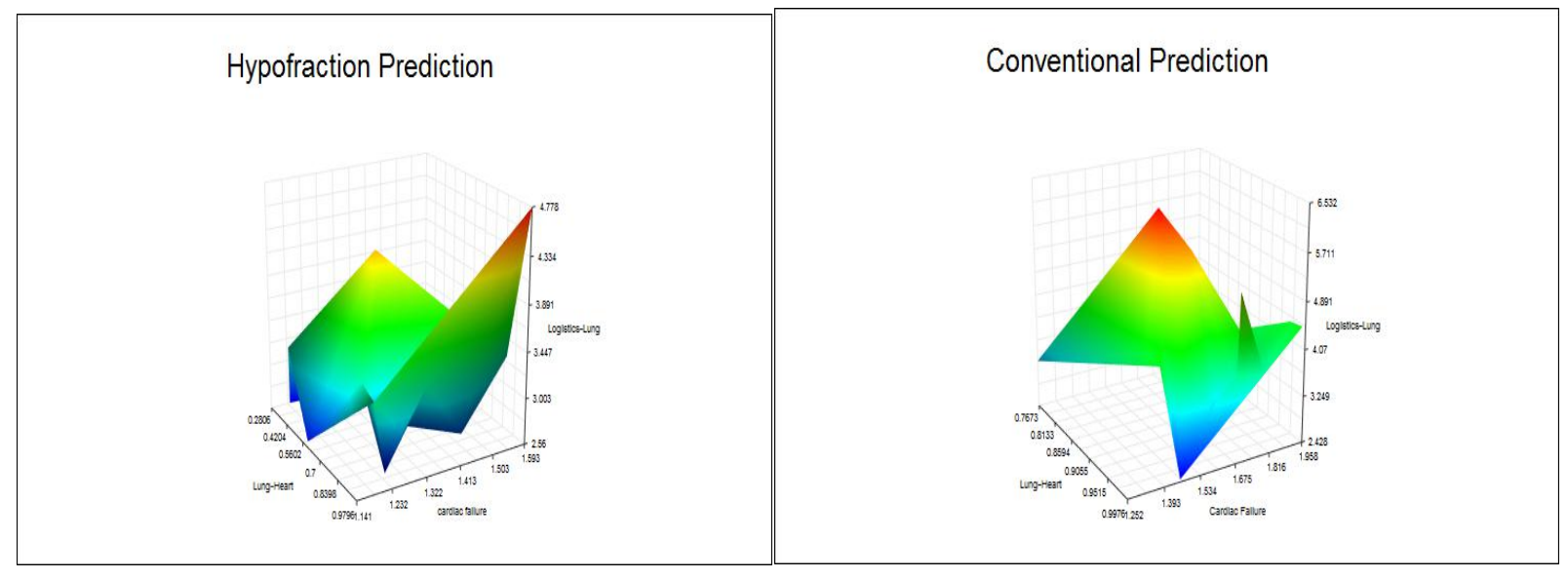

(a)

(b)

Figure 9 a-b: 3D Map of the lung-heart interface in the presence of cardiac failure and lung pneumonitis for hypofraction (a) and conventional (b) treatment of the left breast

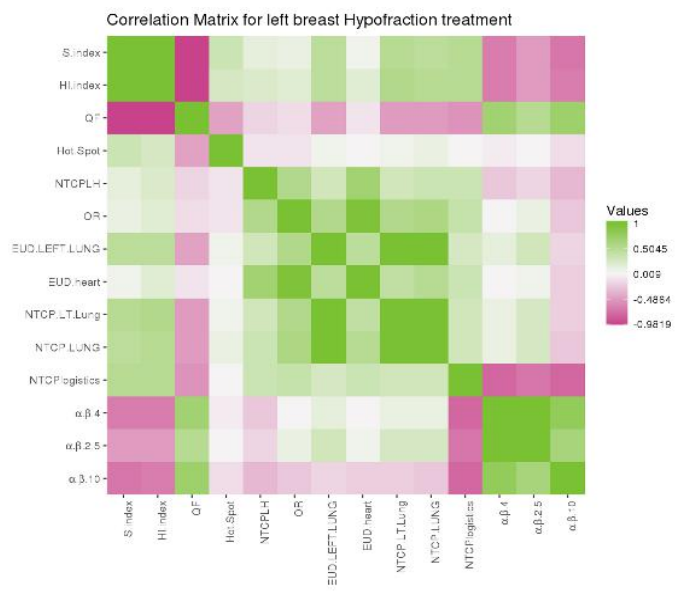

(a)

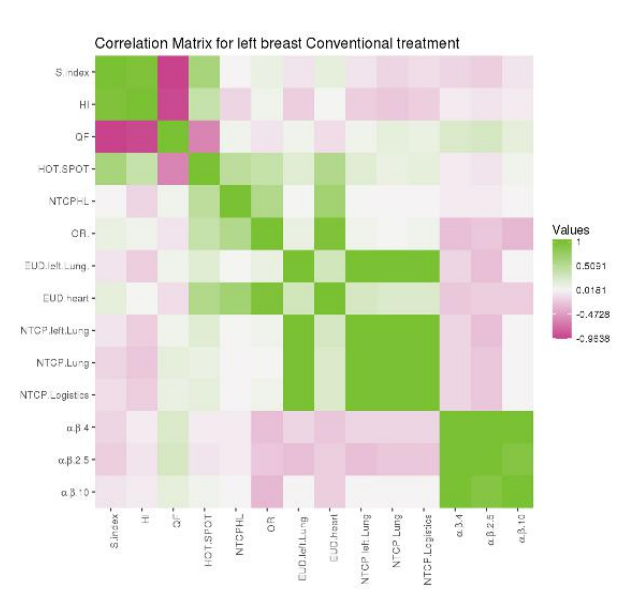

(b)

Figure 10 a-b: The cross-correlation matrixes for the variables linked to hypofraction (a) and to conventional treatment of left breast (b). The color bar represents the Spearman's rank correlation coefficient value

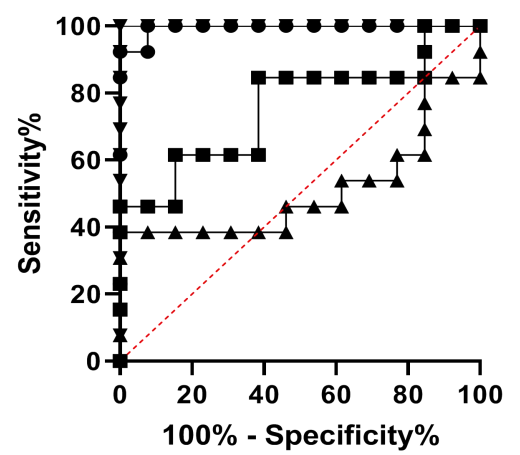

(a)

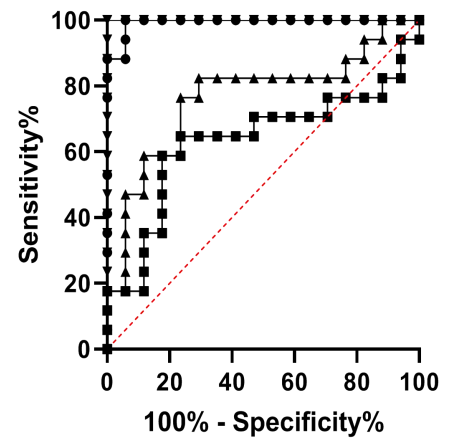

(b)

Figure 11a-b: ROC curve comparison. Logistic regression model vs. NTCP heart-lung $(\Delta)$, EUD left lung $(\nabla)$, NTCP Left lung ( $\bullet$ ), NTCP Total lung (•) for hypofraction (a) and, conventional (b) left breast treatment 


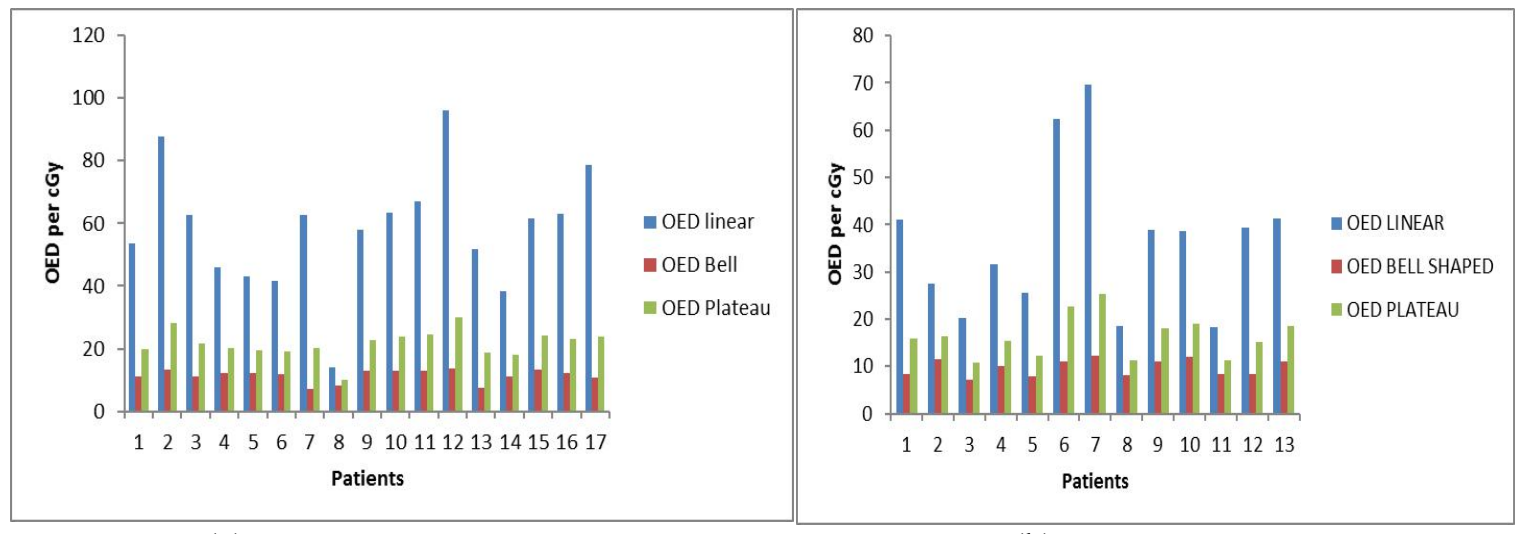

(a)

(b)

Figure 12a-b: Relative risk of secondary cancer per Gy of prescription dose for all patient treatment plans for conventional (a) and hypofraction (b) of left breast for various model

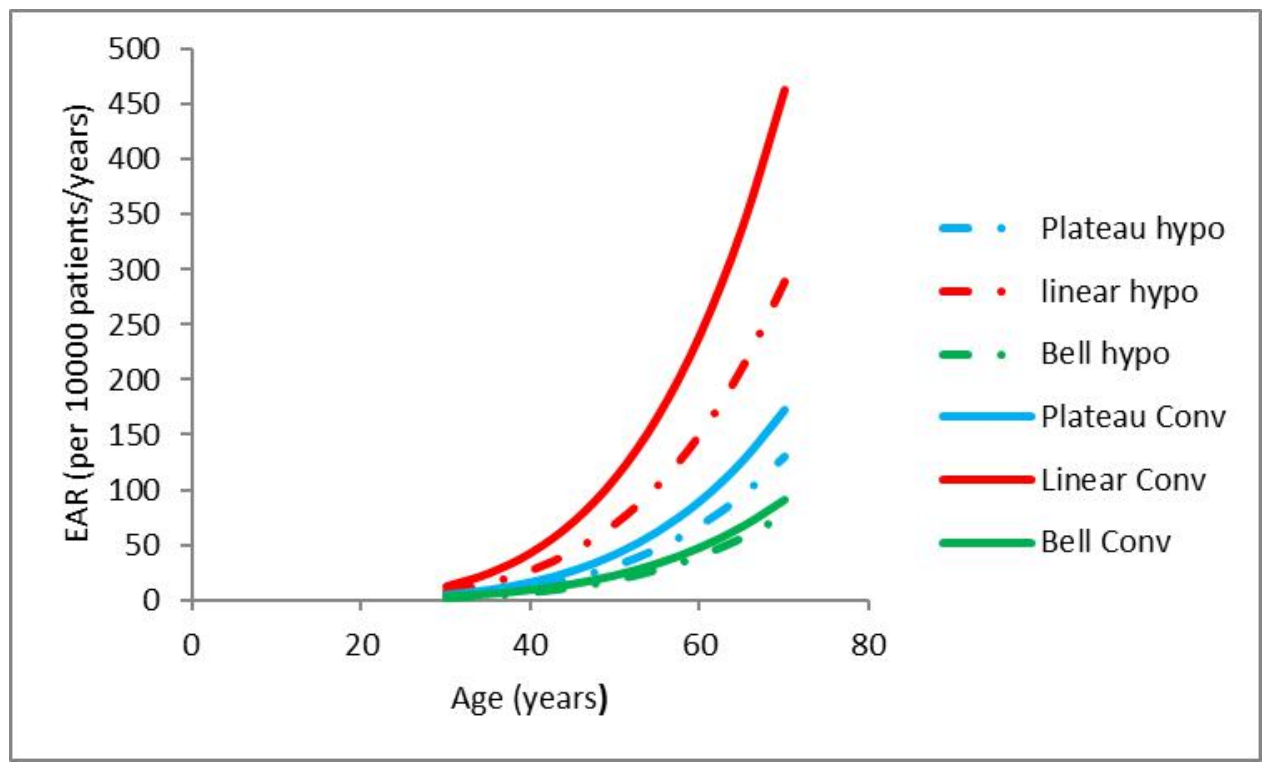

Figure 13: Excess absolute risk of secondary cancer for various risk models (line, conventional treatment; dashed line, hypofraction) for a specific case age at exposure of 30 years and an attained age of 80 years, comparing long-term risks of inducing pneumonitis

\section{Discussion}

Evidence-based observations from several randomized trials and institutional series ${ }^{[25]}$ have shown that after breast conservation surgery, whole breast doses of $42.5 \mathrm{~Gy} / 16$ fractions are as safe and effective as $50 \mathrm{~Gy} / 25$ fractions. Hypofractionated radiation therapy also offers the benefit of streamlining a radiotherapy department's resources with respect to staffing, machines, and patient time. Athas et al. ${ }^{[26]}$ reported that up to $30 \%$ of women in North America do not receive whole breast irradiation (WBI) after breast-conserving surgery for several reasons including age-related morbidity, long travel distance to radiotherapy centers, the personal inconvenience of daily treatments over several weeks, and the high cost of treatment for both patients and the healthcare system. However, hypofractionation is not appropriate for all patients. Complications may occur for patients with large breasts, where a maximum dose of $107 \%$ is not achievable, or patients with implants for augmentation or reconstruction ${ }^{[27]}$. In this study, the mean hot spots were estimated at $106.53 \pm 1.08 \%$ and $109.06 \pm$ $1.66 \%$ for the hypofractionated and conventional treatments respectively. These values compare well 
with those reported by Lazzari et al. ${ }^{[28]}$ who found that $110 \%$ results in good cosmesis.

The main feature of this report is a retrospective analysis of DVH-based metrics on patients who underwent radiotherapy of the left breast. The overall quality of the treatment plans was assessed using DHI, S-index, and QF. The hypofractionated scheme has a better QF in our sample than conventional treatment. However, there is no statistical difference between the treatment regimens in terms of DHI $(\mathrm{p}<0.593)$ or $\mathrm{S}$-index $(\mathrm{p}<0.064)$. When we used $\mathrm{QF}$ in the physical determination, it was sensitive to variations in dose homogeneity and the S-index gradient. However, breast volume did not influence these parameters, in contrast to the report of Karacetin et al., ${ }^{[29]}$ for 3D treatments. The majority of our treatments were performed with the field in field (FiF) technique with a mixed photon beam (18 MV and $6 \mathrm{MV}$ ), which may explain the difference. Some variability can also come from the planning target volume, which has a margin of error ranging from $0 \mathrm{~mm}$ to $30 \mathrm{~mm}$, and the algorithm used for the dose calculation. Herrich et al. ${ }^{[30]}$ showed that the DVH from a FiF-IMRT does not worsen with larger breast volumes. This sentiment is echoed by Aref et al. [31] who found no correlation between breast volume and homogeneity in the conformal planning technique. A breast volume greater than $1800 \mathrm{cc} 25$ will result in more dose inhomogeneity compared to a smaller volume. The mean and standard deviation of breast volume in our study was $1469.74 \pm$ $460.32 \mathrm{cc}$ for conventional treatment and $804.33 \pm$ 303.24 cc for hypofractionated treatment. Consequently, the risk of an overdose to some parts of the breast is low among the patients in our study.

We performed a biological evaluation of the treatments using the LKB and Niermieko models to estimate EUD, NTCP, and TCP. Our EUD values for the left lung and heart respectively were 484.77 $\pm 17.54 \mathrm{cGy}$ and $1217.36 \pm 331.98 \mathrm{cGy}$ under conventional treatment, and $317.24 \pm 157.66 \mathrm{cGy}$ and $640.53 .36 \pm 425.58$ cGy under the hypofractionated treatment. These results compare well with those reported by Adeyemi et al. ${ }^{[32]}$. Also, several studies have reported that the incidence of radiation-induced pneumonitis is in the range of $1 \sim$ $2 \%{ }^{[33-34]}$ similar to our NTCP results of $3.31 \pm$
$0.99 \%$ and $2.45 \pm 0.79 \%$ for the conventional and hypofractionated treatments respectively. The difference between the lung NTCP results for the two regimens is significant, as demonstrated by an unpaired t-test $(\mathrm{p}=0.0133)$. By applying the Lyman model, we found a mean NTCP for pericarditis of $0 \%$ for both regimens, comparable to Utehina et al. ${ }^{[35]}$. The results of TCP calculations using the Poisson method revealed that TCP is very dependent on tissue radiosensitivity. By varying the $\alpha / \beta$ ratio to specific endpoints, TCP decreased with increasing $\alpha / \beta$ for both hypofractionated and conventional treatments. The same observation was made by Deb et al. ${ }^{[36]}$, who suggested that a lower $\alpha$ / $\beta$ ratio leads to a higher TCP, using the Kallman S-model in prostate cases. In our study, there is no significant difference between the two regimens at the two lower $\alpha / \beta$ ratios (for $\alpha / \beta=4$, $p=0.0831$; for $\alpha / \beta=2.5, p=0.6009)$ in contrast with the high $\alpha / \beta$ ratio (for $\alpha / \beta=10, p=0.0399$ ). In addition, we note that for TCP calculations, there is no clear agreement over what $\alpha / \beta$ values to employ as input parameters for the LKB and Poisson models.

After breast conservation therapy, several factors are associated with the TCP calculation. The traditional primary factors are clonogenic cell fraction, dose-response, and the volume effect. In turn, these parameters are influenced by patient heterogeneity, age, differences in surgery, and the extent of the disease. Also, data derived from the DVH depend on the accurate delineation of treatment volumes, an essential element of radiotherapy but also prone to inter-operator variability. The TCP and NTCP values in the study were calculated using radiobiological models, without taking into account the concepts of tumor cell repopulation and reoxygenation during radiotherapy. Hence, the probabilities depicted by TCP and NTCP may differ from the actual clinical situation. On average, left breast irradiation lasts for about 2-3 minutes. On this time scale, it is appropriate to overlook cell repair. For all these reasons, caution is called for when using NTCP and TCP alone to model the toxicity to organs at risk or the actual tumor control probability. However, some important information can still be gleaned from this study. The different radiobiological models 
predicted similar trends, irrespective of the precise TCP or NTCP values calculated.

One of the aims of this study was to model NTCP for heart and lung interaction toxicity. The clinical significance of radiation-induced heart and lung disease is well established, including the complexity of evaluating related risk due to its longterm latency. Through multivariate logistic regression, we built a 4-variable (NTCPLKB, NTCPlogistic, EUD, OR) model with good predictive power, as demonstrated by the ROC curves and their AUC values (AUC $\geqslant 0.754$ ). No difference in performance was found between the hypofractionated and conventional treatments. However, one of the most important aspects to consider in evaluating radiation-induced effects on normal tissue (such as heart and lung, in this study) is that overall risk is an amalgam of complex processes, encompassing multiple biological pathways and systems. In short, we are reporting on the applicability of NTCP models to clinical data as a theoretical tool to aid the prediction of heart and lung toxicity, not a complete and sufficient model in its own right. In short, this paper presents a datadriven and investigative method to model NTCP and a complementary tool to evaluate radiationinduced effects in the cardiopulmonary system, without attempting to model the complicated inherent character of these risks.

Our choice to use Eq.10 and Eq.11 as truth tests to evaluate lung pneumonitis and cardiac failure were based on several factors. First, these equations have stood the test of time as practical guides for predictive toxicity. Second, the lung pneumonitis logistic equation is based on an analysis that combined data from multiple institutions with radiation oncology groups (RTOG 93-11; $\mathrm{n}=324)$, which suggested that lung pneumonitis is best predicted based on mean lung dose. Third, the cardiac failure equation is a combination of several techniques including cardiac Doppler ultrasound, cardiac rhythm, conduction abnormalities with 24-h Holter ECG, 131I-mIBG myocardial scintigraphy, serum brain natriuretic peptide levels at rest, and an exercise test with VO2max measurement on 229 patients. The radiation dose delivered to 7 points in the heart was estimated for all patients who had received radiotherapy.
The secondary cancer risk is more properly estimated for patients who underwent radiotherapy following breast conservation surgery. We estimated secondary cancer risks using the OED and EAR metrics. OED was calculated with three different models: linear, bell-shaped, and plateau. Conventional treatment yielded higher OED values for the left lung under all three models. Further, the OED and EAR values for hypofractionated and conventional treatments under the same model are always significantly different (OED: $p<0.0001$, EAR: $p<0.0001$ ), suggesting that several factors influence the result. Among them, we can count individual differences in radiosensitivity, likely due to genetic differences ${ }^{[37]}$. Also, some reports [38] claim age to be a factor and depict more risk of lung disease for patients less than 30 years old compared to the older breast cancer population ${ }^{[39]}$, similarly to those carrying pre-existing conditions coupled with smoking habits. As some authors [40-41], have pointed out, patients who smoked have a worse pulmonary function and are more susceptible to secondary cancer risk. Radiobiological models are primarily developed for low-dose exposures. The use of mixed photon beams with both high (18 MV) and low (6MV) energy, combined with the field-infield (FiF) treatment technique, may be an additional risk factor for secondary cancer. In particular, when scatter radiation is taken into account, such models can foresee only a fraction of observed secondary malignancies.

There are several limitations associated with the current study. First, it was performed on a small sample of patients and does not take into account several potentially confounding factors including chemotherapy therapy, race, genetic dispositions, and social habits. As a consequence, risk extrapolation to the general population of radiotherapy patients is not evident. Another avenue would be to enrich the sample with long-term survivors of radiotherapy. However, establishing a control population may be hard to achieve.

Second, these results are derived from a model designed for low-dose exposures. As stated above, many confounding factors can play a role in secondary cancer risk. The majority of the models are mechanistic, based mainly on the shape of the dose-response relationships defined in epidemiological cases. For example, for an OED 
calculation based on a plateau response model, as the absorbed dose increases the OED converges to $1 / \delta$. Therefore, the secondary cancer risk depends on the calculated dose as well as on the radiobiological models. The models are dependable and precise from a mathematical standpoint but may not be suitable for our specific sample cases.

Finally, radiotherapy treatments have changed over the years. Nowadays, other techniques are used which render our long-term data a little outdated. In general, different dose distributions in an organ can result in the same OED and trigger the same radiation-induced cancer incidence. Then the EAR as a function of OED and other patient-related cofactors (such as age) could be evaluated via the DVH, with some uncertainties. This is due to the complexity and misunderstanding of secondary cancer induction. In this regard, a report showed that when using treatment plans a precision of $10 \%$ ${ }^{[42]}$ on the prediction should be expected.

\section{Conclusion}

This study has investigated the outcomes of different radiobiological models to calculate NTCP and TCP for hypofraction and conventional left breast treatment in a sample of patients. The results showed a lower NTCP for the heart and warn that TCP is dependent on a choice of radiosensitivity a / $\beta$ parameters. Also, the results suggest that NTCPLKB and NTCP-logistic are interchangeable metrics of lung risk. The outcomes of these models in our sample data were compared with wellestablished multi-institution data. A logistic regression model based on these models has predictive power for lung pneumonitis and pericarditis. Finally, we have shown that secondary cancer incidence can be evaluated via OED and EAR. The shortcomings of this study mainly reside in the scarcity of dosimetric data in the literature and the latency of symptoms. However, there are other ways of reducing cardiac and lung toxicity besides plan optimization and imposing dose constraints to the PTV. For example, one can choose different treatment techniques that include prone positioning and breath holding. It is commonly known that the expected toxicity associated with radiotherapy cannot be calculated from the prescription dose alone [43], but also requires physical models and simple dosimetric descriptors for plan evaluation.

\section{References}

1 Group ST, Bentzen SM, Agrawal RK, Aird EG, Barrett JM, Barrett-Lee PJ, Bentzen SM, Bliss JM, Brown J, Dewar JA, Dobbs HJ, Haviland JS, Hoskin PJ, Hopwood P, Lawton PA, Magee BJ, Mills J, Morgan DA, Owen JR, Simmons S, Sumo G, Sydenham MA, Venables K, Yarnold JR. The UK Standardisation of Breast Radiotherapy (START) Trial B of radiotherapy hypofractionation for treatment of early breast cancer: a randomised trial. Lancet 2008; 371(9618): 1098-1107 DOI: 10.1016/S01406736(08)60348-7

2 Owen JR, Ashton A, Bliss JM, Homewood J, Harper C, Hanson J, Haviland J, Bentzen SM, Yarnold JR. Effect of radiotherapy fraction size on tumor control in patients with earlystage breast cancer after local tumor excision: long-term results of a randomized trial. Lancet Oncol 2006; 7(6): 467-471 [PMID: 16750496 DOI: $\underline{10.1016 / \mathrm{S} 1470-2045(06) 70699-4]}$

3 Whelan TJ, Pignol JP, Levine MN, Julian JA, MacKenzie R, Parpia S, Shelley W, Grimard L, Bowen J, Lukka H, Perera F, Fyles A, Schneider K, Gulavita S, Freeman C. Longterm results of hypofractionated radiation therapy for breast cancer. $\boldsymbol{N}$ Engl J Med 2010; 362(6): 513-520 [PMID: 20147717 DOI: 10.1056/NEJMoa0906260]

4 Group ST, Bentzen SM, Agrawal RK, Aird EG, Barrett JM, Barrett-Lee PJ, Bliss JM, Brown J, Dewar JA, Dobbs HJ, Haviland JS, Hoskin PJ, Hopwood P, Lawton PA, Magee BJ, Mills J, Morgan DA, Owen JR, Simmons S, Sumo G, Sydenham MA, Venables K, Yarnold JR. The UK Standardization of Breast Radiotherapy (START) Trial A of radiotherapy hypofractionation for treatment of early breast cancer: a randomized trial. Lancet Oncol 2008; 9(4): $\quad 331-341 \quad$ DOI: $\quad$ 10.1016/S14702045(08)70077-9

5 Haviland JS, Owen JR, Dewar JA, Agrawal RK, Barrett J, Barrett-Lee PJ, Dobbs HJ, Hopwood P, Lawton PA, Magee BJ, Mills J, 
Simmons S, Sydenham MA, Venables K, Bliss JM, Yarnold JR, Group ST. The UK Standardization of Breast Radiotherapy (START) trials of radiotherapy hypofractionation for treatment of early breast cancer: 10-year follow-up results of two randomized controlled trials. Lancet Oncol 2013; 14(11): 1086-1094 [PMID: 24055415 DOI: 10.1016/S1470-2045(13)70386-3]

6 Jagsi R, Griffith KA, Boike TP, Walker E, Nurushev T, Grills IS, Moran JM, Feng M, Hayman J, Pierce LJ. Differences in the Acute Toxic Effects of Breast Radiotherapy by Fractionation Schedule: Comparative Analysis of Physician-Assessed and Patient-Reported Outcomes in a Large Multicenter Cohort. JAMA Oncol 2015; 1(7): 918-930 [PMID: 26247417 DOI: $10.1001 /$ jamaoncol.2015.2590]

7 Shaitelman SF, Schlembach PJ, Arzu I, Ballo M, Bloom ES, Buchholz D, Chronowski GM, Dvorak T, Grade E, Hoffman KE, Kelly P, Ludwig M, Perkins GH, Reed V, Shah S, Stauder MC, Strom EA, Tereffe W, Woodward WA, Ensor J, Baumann D, Thompson AM, Amaya D, Davis T, Guerra W, Hamblin L, Hortobagyi G, Hunt KK, Buchholz TA, Smith BD. Acute and Short-term Toxic Effects of Conventionally Fractionated vs Hypofractionated Whole-Breast Irradiation: A Randomized Clinical Trial. JAMA Oncol 2015; 1(7): $931-941 \quad$ DOI: $\underline{10.1001 / j a m a o n c o l .2015 .2666}$

8 Story M, Kodym R, Saha D. Exploring the possibility of unique molecular, biological, and tissue effects with hypofractionated radiotherapy. Semin Radiat Oncol 2008; 18(4): 244-248 [PMID: 18725111 DOI: 10.1016/j.semradonc.2008.04.006]

9 Group ST, Bentzen SM, Agrawal RK, Aird EG, Barrett JM, Barrett-Lee PJ, Bliss JM, Brown J, Dewar JA, Dobbs HJ, Haviland JS, Hoskin PJ, Hopwood P, Lawton PA, Magee BJ, Mills J, Morgan DA, Owen JR, Simmons S, Sumo G, Sydenham MA, Venables K, Yarnold JR. The UK Standardisation of Breast Radiotherapy (START) Trial A of radiotherapy hypofractionation for treatment of early breast cancer: a randomized trial. Lancet Oncol 2008;
9(4): $\quad 331-341 \quad$ DOI: $\quad$ 10.1016/S14702045(08)70077-9

10 Group ST, Bentzen SM, Agrawal RK, Aird EG, Barrett JM, Barrett-Lee PJ, Bentzen SM, Bliss JM, Brown J, Dewar JA, Dobbs HJ, Haviland JS, Hoskin PJ, Hopwood P, Lawton PA, Magee BJ, Mills J, Morgan DA, Owen JR, Simmons S, Sumo G, Sydenham MA, Venables K, Yarnold JR. The UK Standardization of Breast Radiotherapy (START) Trial B of radiotherapy hypofractionation for treatment of early breast cancer: a randomized trial. Lancet 2008; 371(9618): 1098-1107 DOI: $\underline{10.1016 / \mathrm{S} 0140-}$ $\underline{6736(08) 60348-7}$

11 Whelan TJ, Kim DH, Sussman J. Clinical experience using hypofractionated radiation schedules in breast cancer. Semin Radiat Oncol 2008; 18(4): 257-264 [PMID: 18725113 DOI: 10.1016/j.semradonc.2008.04.008]

12 Sanchez-Nieto B, Nahum AE. BIOPLAN: software for the biological evaluation of. Radiotherapy treatment plans. Med Dosim 2000; 25(2): 71-76 [PMID: 10856684]

13 Yoon M, Park SY, Shin D, Lee SB, Pyo HR, Kim DY, Cho KH. A new homogeneity index based on statistical analysis of the dose-volume histogram. J Appl Clin Med Phys 2007; 8(2): 9-17 DOI: 10.1120/jacmp. v8i2.2390

14 Pyakuryal A, Myint WK, Gopalakrishnan M, Jang S, Logemann JA, Mittal BB. A computational tool for the efficient analysis of dose-volume histograms from radiation therapy treatment plans. J Appl Clin Med Phys 2010; 11(1): 3013

15 Niemierko A, Goitein M. Modeling of normal tissue response to radiation: the critical volume model. Int J Radiat Oncol Biol Phys 1993; 25(1): 135-145 [PMID: 8416870 DOI: 10.1016/0360-3016(93)90156-p]

16 Burman C, Kutcher GJ, Emami B, Goitein M. Fitting of normal tissue tolerance data to an analytic function. Int $\boldsymbol{J}$ Radiat Oncol Biol Phys 1991; 21(1): 123-135 [PMID: 2032883 DOI: 10.1016/0360-3016(91)90172-z]

17 Kutcher GJ, Burman C: Calculation of complication probability factors for nonuniform normal tissue irradiation: the effective 
volume method. Int J Radiat Oncol Biol Phys 1989, 16:1623 - 1630.

18 Kutcher GJ, Burman C. Calculation of complication probability factors for nonuniform normal tissue irradiation: the effective volume method. Int J Radiat Oncol Biol Phys 1989; 16(6): 1623-1630 [PMID: 2722599 DOI: 10.1016/0360-3016(89)90972-3]

19 Marks LB, Bentzen SM, Deasy JO, Kong FM, Bradley JD, Vogelius IS, El Naqa I, Hubbs JL, Lebesque JV, Timmerman RD, Martel MK, Jackson A. Radiation dose-volume effects in the lung. Int J Radiat Oncol Biol Phys 2010; 76(3 Suppl): S70-76 DOI: 10.1016/j.ijrobp.2009.06.091

20 Guldner L, Haddy N, Pein F, Diallo I, Shamsaldin A, Dahan M, Lebidois J, Merlet P, Villain E, Sidi D, Sakiroglu O, Hartmann O, Leftakopoulos D, de Vathaire F. Radiation dose and long-term risk of cardiac pathology following radiotherapy and anthracyclin for a childhood cancer. Radiother Oncol 2006; 81(1): $\quad 47-56 \quad$ DOI: 10.1016/j.radonc.2006.08.020

21 Cella L, Palma G, Deasy JO, et al. Complication Probability Models for Radiation-Induced Heart Valvular Dysfunction: Do Heart-Lung Interactions Play a Role? Amendola R, ed. PLoS ONE. 2014; 9(10) https://doi.org/10.1371/journal.pone.0111753

22 ICRP Publication 103. Ann. ICRP 2007, 37, 1332.33

23 BEIR VII: Health Risks from Exposure to Low Level of Ionizing Radiation; National Research Council. The National Academies: Washington, DC, USA, 2006.

24 Schneider U, Sumila M, Robotka J. Sitespecific dose-response relationships for cancer induction from the combined Japanese Abomb and Hodgkin cohorts for doses relevant to radiotherapy. Theor Biol Med Model 2011; 8: 27 DOI: 10.1186/1742-4682-8-27

25 The START Trialists' Group: The UK Standardization of Breast Radiotherapy (START) Trial A of radiotherapy hypofractionation for treatment of early breast cancer: a randomized trial, Lancet Oncol. 2008 Apr 1; 9(4): 331-341.
26 Athas WF, Adams-Cameron M, Hunt WC, Amir-Fazli A, Key CR. Travel distance to radiation therapy and receipt of radiotherapy following breast-conserving surgery. $\boldsymbol{J}$ Natl Cancer Inst 2000; 92(3): 269-271 [PMID: 10655446 DOI: 10.1093/jnci/92.3.269]

27 Koulis TA, Phan T, Olivotto IA. Hypofractionated whole breast radiotherapy: current perspectives. Breast Cancer (Dove Med Press) 2015; 7: 363-370 DOI: 10.2147/BCTT.S81710

28 Lazzari G, Terlizzi A, Della Vittoria Scarpati G, Perri F, De Chiara V, Turi B, Silvano G. Predictive parameters in hypofractionated whole-breast 3D conformal radiotherapy according to the Ontario Canadian trial. Onco Targets Ther 2017; 10: 1835-1842 DOI: 10.2147/OTT.S127833

29 Didem Karacetin., et al. "Dosimetric Comparison of Dynamic IMRT, Field-in-Field IMRT and 3D-CRT in Left-Sided Breast Cancer after Breast-Conserving Surgery". Acta Scientific Cancer Biology 2.5 (2018): 06-10.

30 Herrick JS, Neill CJ, Rosser PF. A comprehensive clinical 3-dimensional dosimetric analysis of forward planned IMRT and conventional wedge planned techniques for intact breast radiotherapy. Med Dosim 2008; 33(1): 62-70 [PMID: 18262125 DOI: 10.1016/j.meddos.2007.06.001]

31 Aref A, Thornton D, Youssef E, He T, TekyiMensah S, Denton L, Ezzell G. Dosimetric improvements following 3D planning of tangential breast irradiation. Int $\boldsymbol{J}$ Radiat Oncol Biol Phys 2000; 48(5): 1569-1574 [PMID: 11121663 DOI: $10.1016 / \mathrm{s} 0360-$ 3016(00)00808-7]

32 Adeyemi OF, Osahon OD, Okungbowa GE (2017) Evaluation and Optimization of treatment Plans for Post Mastectomy Patients using Radiobiological Models in a LowResource Center, Nigeria. J Cancer Biol Res 5(3): 1107.

33 Das IJ, Cheng EC, Schultz DJ, Harris EE, Solin LJ. Radiation pneumonitis in megavoltage beam treatment of breast cancer. Int $\boldsymbol{J}$ Radiat Oncol Biol Phys 2001; 51:245-246

34 Taghian AG, Assaad SI, Niemierko A, Kuter I, Younger J, Schoenthaler R, Roche M, Powell 
SN. Risk of pneumonitis in breast cancer patients treated with radiation therapy and combination chemotherapy with paclitaxel. $\boldsymbol{J}$ Natl Cancer Inst 2001; 93(23): 1806-1811 DOI: $10.1093 /$ jnci/93.23.1806

35 Utehina O, Popovs S, Purina D, Slosberga I, Vevere I, Emzins D, Berzins J, Valuckas KP, Janulionis E, Miller A. Analysis of cardiac and pulmonary complication probabilities after radiation therapy for patients with early-stage breast cancer. Medicina (Kaunas) 2009; 45(4): 276-285 [PMID: 19423958]

36 Deb P, Fielding A. Radiobiological model comparison of 3D conformal radiotherapy and IMRT plans for the treatment of prostate cancer. Australas Phys Eng Sci Med 2009; 32(2): 51-61 [PMID: 19623855]

37 Turesson I, Nyman J, Holmberg E, Oden A. Prognostic factors for acute and late skin reactions in radiotherapy patients. Int $\boldsymbol{J}$ Radiat Oncol Biol Phys 1996; 36(5): 1065-1075 DOI: 10.1016/s0360-3016(96)00426-9

38 Jensen BV, Carlsen NL, Nissen NI. Influence of age and duration of follow-up on lung function after combined chemotherapy for Hodgkin's disease. Eur Respir J 1990; 3(10): 1140-1145 [PMID: 2090476]
39 Lind PA, Svane G, Gagliardi G, Svensson C. Abnormalities by pulmonary regions studied with computer tomography following local or local-regional radiotherapy for breast cancer. Int J Radiat Oncol Biol Phys 1999; 43(3): 489-496 DOI: 10.1016/s0360-3016(98)00414$\underline{3}$

40 Hermann T SJ, Molls M. Radiation Pneumopathy. Experimental and clinical data. In: Late sequelae in oncology. In: Dunst J und Sauer $R$ H, editor, Berlin, Heidelberg, New York 1995. p. 135-40.

41 Knudson RJ, Kaltenborn WT, Burrows B. The effects of cigarette smoking and smoking cessation on the carbon monoxide diffusing capacity of the lung in asymptomatic subjects. Am Rev Respir Dis 1989; 140(3): 645-651 DOI: $\underline{10.1164 / \text { ajrccm/140.3.645 }}$

42 Nguyen J, Moteabbed M, Paganetti $H$. Assessment of uncertainties in radiationinduced cancer risk predictions at clinically relevant doses. Med Phys 2015; 42(1): 81-89 DOI: $10.1118 / 1.4903272$

43 Kong FM, Hayman JA, Griffith KA, Kalemkerian GP, Arenberg D, Lyons S, Turrisi A, Lichter A, Fraass B, Eisbruch A, Lawrence TS, Ten Haken RK. Final toxicity results of a radiation-dose escalation study in patients with non-small-cell lung cancer (NSCLC): predictors for radiation pneumonitis and fibrosis. Int J Radiat Oncol Biol Phys 2006; 65(4): 1075-1086 DOI: 10.1016/j.ijrobp.2006.01.051] 